
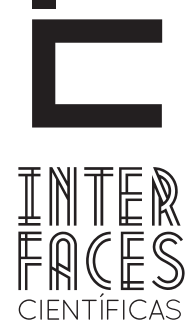

SAÚDE E AMBIENTE

ISSN IMPRESSO 2316-3313

E-ISSN 2316-3798

DOI - 10.17564/2316-3798.2017v6n1p83-94

\title{
VIVÊNCIAS DE PORTADORES DE TUBERCULOSE E IMPORTÂNCIA DA FAMÍLIA À ADESÃO TERAPÊUTICA
}

EXPERIENCES OF PATIENTS WITH TUBERCULOSIS AND THE IMPORTANCE OF FAMILY FOR ADHERENCE TO TREATMENT. EXPERIENCIAS DE LOS PACIENTES CON TUBERCULOSIS Y LA IMPORTANCIA DE LA FAMILIA PARA LA ADHERENCIA AL TRATAMIENTO

Ayanne Nathalya Muniz da Silva

Adalmira Batista Lima ${ }^{3}$

José Cleston Alves Camboim ${ }^{5}$
Milena Nunes Alves de Sousa

Maria Cecília Lopes da Silva ${ }^{4}$

Francisca Elidivânia de Farias Camboim

\section{RESUMO}

Este trabalho teve por objetivo analisar as vivências de portadores de tuberculose frente à descoberta da patologia e a importância da família para a adesão ao tratamento. Estudo do tipo descritivo, de campo com abordagem qualitativa, realizada nas Unidades de Saúde da Família da cidade de Patos-PB. Participaram do estudo nove portadores de tuberculose, obedecendo aos critérios de inclusão da pesquisa. Os dados foram analisados à luz da literatura pertinente e o projeto foi aprovado pelo Comitê de Ética em Pesquisa das Faculdades Integradas de Patos. 0 portador de tuberculose ainda enfrenta preconceitos advindos da sociedade e de familiares em relação à patologia, além das mudanças que ocorrem de forma significativa em sua vida, podendo acarretar inclusive, nos aspectos emocionais de cada um. 0 apoio familiar e social é um dos fatores primordiais para êxito do tratamento e a equipe de saúde exerce papel fundamental no esclarecimento dos cuidados do portador de Tuberculose (TB).

\section{PALAVRAS-CHAVE}

Tuberculose. Relações Familiares. Terapêutica. 


\section{ABSTRACT}

This work aimed to analyze the experiences of patients with tuberculosis forward to the discovery of the disease and the importance of family for adherence to treatment. Descriptive study, field with a qualitative approach, held in the Family Health Units of the city of Patos- PB. The study included 09 patients with tuberculosis, according to the research inclusion criteria. Data were analyzed in the light of the relevant literature, and the project was approved by the Research Ethics Committee of the International College of ducks. The carrier of tuberculosis still faces very arising prejudice of society and family regarding the disease, in addition to changes that occur significantly in your life, which may cause even the emotional aspects of each. The family and social support is a primary factor for successful treatment, and the health team plays a fundamental role in resolving the TB carrier care.

\section{KEYWORDS}

Tuberculosis. Family relationships. Therapy.

\section{RESUMEN}

Este trabajo tuvo por objetivo analizar las experiencias de los pacientes con tuberculosis con interés el descubrimiento de la enfermedad y la importancia de la familia para unirse al tratamiento. Estudio descriptivo, de campo con un enfoque cualitativo, realizado en las unidades de salud de la familia de la ciudad de Patos- PB. El estudio incluyó a 09 pacientes con tuberculosis, de acuerdo con los criterios de inclusión de investigación. Los datos fueron analizados a la luz de la literatura relevante, y el proyecto fue aprobado por el Comitê de Ética em Pesquisa das Faculdades Integradas de Patos. El portador de la tuberculosis aún enfrenta prejuicios por parte de la sociedad y la familia con respecto a la enfermedad, además de los cambios que se producen de manera significativa en su vida, que pueden causar, incluso a los aspectos emocionales de cada uno. La familia y el apoyo social es un factor primordial para el éxito del tratamiento, y el equipo de salud tiene un papel fundamental en la resolución de los cuidados de soporte al portador de tuberculosis.

\section{PALABRAS CLAVE}

Tuberculosis; Las relaciones familiares; Terapia. 


\section{INTRODUÇ̄̃̃O}

A Tuberculose (TB) ainda é considerada como um dos grandes problemas de saúde pública, não somente no Brasil, mas, mundialmente. Sendo caracterizada pelo reflexo da desigualdade social, devido sua incidência ser principalmente em países com piores condições socioeconômicas, aliadas a um dos principais desafiadores no controle da disseminação da tuberculose que é a vigilância, a desorganização dos serviços de saúde e o preconceito que a sociedade apresenta (LACERDA et al., 2014).

O número de casos da TB acomete intensamente populações que vivem em aglomerados urbanos, indivíduos que vivem em condições precárias de habitação, população carcerária e o índice de mortalidade aumenta quando se está envolvido a coinfecção de tuberculose/ HIV (HIJAR; CAMPOS; FEITOSA, 2013).

Desde seu surgimento, a tuberculose teve diversas denominações como: Tísica, peste branca, demônio do rei, doença mesentérica, mal de Pott, escrófula, mas após diversos estudos, em 1882, Robert Kock, médico alemão, bacteriologista anunciava a descoberta do bacilo causador da tuberculose, que em sua homenagem é também chamada de Bacilo de Kock (BK) (MACIEL et al., 2012).

A tuberculose é uma doença infecto-contagiosa causada pelo Mycobacterium tuberculosis, também conhecido por Bacilo de Kock, é predominante no sexo masculino, sua transmissão ocorre por meio das vias áreas de uma pessoa contaminada para outra sadia, é uma doença tão antiga quanto à humanidade e dispõe de tratamento eficaz e gratuito desde a década de 1960 (BRASIL, 2011; SILVA et al., 2016). A Organização Mundial de Saúde (OMS) revela que a taxa de incidência da tuberculose vem diminuindo desde 2002 em 1,3\% ao ano, 22 países são responsáveis por $81 \%$ de todos os casos de tuberculose no mundo, sendo os cinco primeiros a Îndia, China, África do sul, Paquistão e Indonésia, o Brasil está no $17^{\circ}$ lugar (BRASIL, 2011).

Estudo aponta as principais situações que interferem no sucesso do controle da TB que são: a preca- riedade dos serviços para assistência ao doente ou ao indivíduo com suspeita de TB, a dificuldade de acesso aos meios de transporte e a rede de saúde pública, falta de recursos para alimentar-se e para dar continuidade ao tratamento, essas situações comprometem o diagnóstico precoce e prejudica a continuidade do tratamento (LEMOS; FEIJÃO; GALVÃO, 2013).

Um dos grandes problemas do Brasil é em relação ao desenvolvimento social do país, devido ao estado de pobreza, juntamente com as condições sanitárias, a desorganização e deficiência do setor de saúde. A região nordeste por sua vez, devido ao desenvolvimento socioeconômico dificulta ao extremo as ações de prevenção e controle da tuberculose. Com o surgimento da AIDS, o perfil epidemiológico da tuberculose mudou quase por completo, pois uma patologia agrava a progressão da outra enfermidade, aumentando assim, a incidência de morbimortalidade em todo o mundo (CLEMENTINO et al., 2011).

A Estratégia Saúde da Família (ESF) tem um papel importantíssimo no controle, detecção precoce e tratamento dos indivíduos infectados ou com suspeita de TB, como: realizar a busca ativa dos comunicantes junto aos Agentes Comunitários de Saúde (ACS), os profissionais sensibilizados com a causa devem implementar ações de promoção a saúde a esses indivíduos, os gestores devem viabilizar políticas públicas centradas e favorecer uma melhoria de qualidade de vida a essas pessoas para que restabeleçam a imunidade e assim sintam-se fortalecidas a dar continuidade ao tratamento que por sua vez debilita muito o organismo (MARQUIEVIZ et al., 2013).

Além da dedicação dos profissionais e as medidas de controle e tratamento que é totalmente gratuito, busca-se compreender qual a questão que leva o indivíduo a não querer ou dar continuidade ao tratamento, abandonar, qual a sua percepção diante a doença no âmbito familiar e social, mesmo compreendendo que muitas vezes a doença surge no auge de sua vida e que afeta não só sua vivência, mas a de todos que o rodeiam. 
O motivo da escolha do tema se deu a partir do interesse em compreender o sentimento dos portadores de TB em relação à patologia e o estigma social existente.

A pesquisa teve como objetivo analisar as vivências de portadores de tuberculose frente à descoberta da patologia e a importância da família para a adesão ao tratamento.

\section{MATERIAL E MÉTODOS}

Trata-se de uma pesquisa de campo, descritiva com abordagem qualitativa, a qual ocorreu nas Estratégias de saúde da família, localizados no município de Patos-PB, somando 27 unidades. 0 estudo compreendeu um universo de nove portadores de tuberculose na faixa etária compreendida de 24 a 54 anos de idade, prevalecendo portadores de TB com idade entre 49 a 54 anos (33\%), do sexo masculino (89\%), solteiros (44\%), agricultores (33\%) com uma renda mensal de aproximadamente 2 salários mínimos (67\%), obedecendo a apenas um critério de inclusão: Ter idade superior a 18 anos de idade. Foram excluídas as pessoas que não estiveram presentes no ato da entrevista. 0 período das entrevistas ocorreu entre os meses de setembro e outubro de 2015, a coleta se deu na própria residência dos participantes do estudo, encerrando-se frente à repetição de respostas dos portadores de tuberculose.

Aplicou-se um instrumento de pesquisa por meio de um roteiro de entrevista estruturado, elaborado pelos próprios autores, contendo questões relacionadas ao objetivo do estudo: Como aconteceu/acontece o apoio familiar em relação a sua doença? Fale sobre seus medos em relação à Tuberculose. Fale sobre suas dificuldades em realizar o seu tratamento. Fale sobre o preconceito da sociedade frente à tuberculose. Quais as mudanças que ocorreram em sua vida depois do diagnóstico da doença?

A análise das entrevistas iniciou-se por meio de uma transcrição. O segundo momento correspondeu à identificação de temas, nos discursos, sobre as questões elaboradas. Depois, os textos foram de- compostos e organizados em blocos de significados por coincidência/divergência temática. Para manter o anonimato dos participantes, as entrevistas foram citadas por nome de pássaros, tais como: Bem te vi, Beija flor, Andorinha, Pombo, Galo de campina, Pica pau, Pardal, Canário, Azulão. Para analisar os dados coletados, houve a leitura das entrevistas, as falas dos entrevistados foram consideradas com base na Análise Temática por meio desta foi possível descobrir as categorias que compõem a comunicação, associando as significações expressas pelos sujeitos do estudo (BARDIN, 2011).

Assim, a Análise Temática transcorreu pelas seguintes etapas: na primeira etapa, ocorreu a compreensão dos dados coletados por meio da leitura; na segunda, pelas falas das entrevistadas começaram a emergir categorias temáticas; na terceira, a análise realizada é comparada com outros estudos que abordam o assunto; na última etapa aconteceu a descoberta das categorias, as quais foram interpretadas e discutidas. Diante da análise das falas dos portadores de tuberculose nas entrevistas, emergiram três categorias: Vivência de portadores de tuberculose frente à descoberta da patologia, $\mathrm{A}$ importância do contexto familiar na promoção da qualidade de vida ao portador de tuberculose e, 0 reflexo do preconceito social na vida do portador de tuberculose.

0 estudo, atendendo a Resolução nº 466/12 do Conselho Nacional de Saúde (BRASIL, 2013), foi realizado segundo os princípios éticos de pesquisa em humanos, por meio da aprovação do Comitê de Ética em Pesquisa das Faculdades Integradas de Patos, conforme protocolo CAAE: 45307215.0.0000.5181, parecer $\mathrm{n}^{\circ} 1.149 .861 / 2015$.

\section{RESULTADOS E DISCUSSÃO}

\subsection{VIVÊNCIA DE PORTADORES DE TUBERCULOSE FRENTE À DESCOBERTA DA PATOLOGIA}

Na maioria dos casos a tuberculose é transmitida pelo contato direto com o portador bacilífero e os há- 
bitos de vida que o portador é acostumado a exercer diariamente muitas vezes comprometem a eficácia do tratamento adequado para obter a cura (LACERDA et al., 2014). É importante que o portador de TB tenha uma percepção do valor da sua posição na vida, quanto a sua saúde no presente, assim como sua própria saúde no futuro. Compreender que a doença não afeta apenas a si própria, afetando concomitantemente a família, a comunidade e o ambiente profissional. Por isso faz-se necessário abandonar alguns hábitos de vida para adquirir a cura e o portador voltar à convivência com o grupo familiar e a comunidade como um todo, sem o estigma e a preocupação de estar transmitindo a doença.

Acredita-se que quando o portador adquire outros hábitos de vida positivo em relação a sua doença, ele muda do grau de desinformação e dependendo do próprio interesse de querer mudar e/ou agir ele pode tornar-se atuante no processo de transformação, melhorando o processo saúde, doença e sua qualidade de vida (BRASIL, 2011; SOUSA; SILVA, 2013). A seguir, estão evidenciados os discursos dos participantes do estudo, frente a sua vivência quando da descoberta da tuberculose:

[...] Tive que parar de fumar, beber e trabalhar. (ANDORINHA).

[...] Não fumo mais, nem bebo, não pego em poeira e evito ficar em lugares fechados. (POMBO).

[...] Parei de andar na poeira, sol quente, tive que ficar mais em casa, parei de fumar e beber. (GALO DE CAMPINA).

[...] Continua quase da mesma forma, só tive que parar de fumar, beber e evitar poeiras. (CANÁRIO).

[...] Não posso sair muito de casa, não posso beber mais e nem fumar. (BEM TI VI).

[...] Parei mais de sair de casa no início do tratamento, deixei mais de beber e tento não ficar pegando em poeiras. (AZULÃO).

No presente estudo todos os portadores de tuberculose tiveram que mudar e/ou restringir alguns hábi- tos comuns do dia a dia, tendo como principal objetivo a obtenção da cura. A família é a primeira instância do cuidado em saúde, constituindo o papel que tem mais significância na tomada de decisões dos portadores de tuberculose, no que diz respeito ao seu processo saúde/doença (FREITAS et al., 2012).

Para os autores citados, o ambiente familiar tem papel fundamental desde a suspeita quanto à continuidade do tratamento, pois é por meio do apoio dos integrantes da família que o portador de tuberculose irá encontrar forças para enfrentar a luta diária com os efeitos adversos que as medicações causam, assim, como enfrentar o preconceito que a sociedade poderá apresentar. Uma das principais dificuldades em diminuir a incidência ou mesmo erradicar a doença está relacionada com o aumento da pobreza, condições precárias de habitação, alcoolismo e outros hábitos viciosos de vida e o estigma do preconceito.

A qualidade de vida do portador de TB irá depender de suas decisões no que diz respeito a sua própria saúde, os cuidados que terão que ter, os vícios que terão de deixar, tendo por finalidade aumentar a imunidade e diminuir os riscos de resistência medicamentosa. A equipe de saúde tem um papel importantíssimo nesse cuidado, por que deverá repassar todas as informações necessárias para o portador e a família, que apesar de ser uma patologia grave, tem tratamento e cura se realizado adequadamente. É importante lembrar que durante e depois do tratamento o portador não deve ingerir bebidas alcoólicas e nem fumar, para evitar assim uma lesão no fígado ou diminuição do efeito desejado da medicação (NOGUEIRA et al., 2011).

0 desconhecimento em face da doença, antes ou após o diagnóstico e esclarecimento sobre o tratamento da tuberculose, muitos portadores guardam para si seus medos, angustias e tentam esconder de amigos e familiares seus sentimentos mais íntimos, a fim de evitar situações constrangedoras. Como para qualquer patologia agravante que precise de cuidados rigorosos e muitas vezes afastamento de pessoas queridas, o portador de tuberculose também tem seus medos e preocupações em relação a sua vida em diante. 
Contudo, para o portador de tuberculose, que ao adoecer as limitações sentidas já não o condenam a uma morte social, tendo em vista que o resultado do tratamento certo tem resposta $100 \%$ de cura (ROCHA; CRUZ; FERMINO, 2013). Mediante análise, foi possível identificar alguns fatores que contribuem para despertar os medos e angustias dos portadores de tuberculose.

[...] Medo de nada, mais tive um pouco de ansiedade e receio da internação hospitalar. (ANDORINHA).

[...] Tive medo antes de saber o que era, por que pensava ser outra doença mais grave, mais depois de saber que era tuberculose fiquei mais tranquilo por que sabia que podia combater. (GALO DE CAMPINA).

[...] Tive medo do que as pessoas iam pensar, de não ficar bom ou adoecer outras pessoas. (AZULÃO).

A tuberculose é uma patologia que exige muita dedicação do portador, uma vez firmado o diagnóstico, muitos terão que se habituar a mudanças no estilo de vida, o que gera insegurança, tensão emocional e medo. A preocupação com a possibilidade de contaminar outras pessoas faz com que os tuberculínicos se isolem ou se sintam excluídas mesmo antes desse fato acontecer (FARIAS et al., 2013).

Para muitos portadores de tuberculose a doença gera inúmeras interrogações, questionamentos e para enfrentar um diagnóstico positivo na maioria das vezes significa estar diante de manifestações preconceituosas que familiares, amigos e a sociedade poderão apresentar, assim como medo e rejeição advindos das pessoas que o portador mais precisa de apoio, que são seus familiares e amigos. Assim, a satisfação com a vida e a sensação de bem estar pode ser afetada após o diagnóstico da doença crônica como a tuberculose (ROCHA; CRUZ; FERMINO, 2013).

Vale ressaltar que na atualidade a família não é apenas aquela constituída por laços de consanguinidade, mas sim, são todos aqueles que estão próximos a ela e que influenciam direta, negativa ou positivamente sobre os membros da família, sendo assim, a família tem um papel importante desde o diagnóstico da doença até o portador receber alta por cura (SOUSA; SILVA; MEIRELLES, 2010). Faz-se necessário que o portador encontre na família apoio emocional e psicológico para conseguir enfrentar a doença que debilita muito o organismo, assim a qualidade de vida dos portadores de tuberculose é eminentemente humana que é aproximada na satisfação encontrada no ambiente familiar, social, amoroso e ambiental (ROCHA; CRUZ; FERMINO, 2013).

0 portador de tuberculose enfrenta diversos desafios de vida, começando desde a situação socioeconômica desfavorável, que na maior parte dos casos fazem-se presente, até as dificuldades de acesso ao serviço de saúde, tudo isso pode ser favorável para disseminação da tuberculose (OLIVEIRA et al., 2011).

O tuberculínico, após o diagnóstico positivo da cultura de escarro, inicia o tratamento com quatro medicações que são ingeridas associadamente, as quais causam no portador diversos efeitos adversos e desagradáveis (BRASIL, 2011), o que pode ser motivo de desistência do tratamento, uma vez que a equipe de saúde não tem o hábito de explicar os efeitos que a medicação poderá causar no organismo e o portador, às vezes, não tem consciência da adesão ao tratamento e sua evolução.

Apesar de ter tratamento $100 \%$ eficaz existem fatores que intervêm nos resultados como a resistência medicamentosa e o tratamento incompleto (PINHEIRO et al., 2013). As medicações causam diversos efeitos adversos e para completar o tratamento o portador terá de enfrentá-las. No presente estudo podem-se avaliar algumas das dificuldades enfrentadas pelos portadores de tuberculose durante seu tratamento.

[...] Não. Só as medicações que são muito fortes e às vezes tira o apetite (BEIJA - FLOR).

[...] Sinto cansaço e falta de apetite (PARDAL).

[...] Não, apesar das reações que os medicamentos causam, sei que é preciso (BEM TI VI).

0 tratamento é de duração longa, contudo, após o início da terapêutica o portador já apresenta me- 
Ihora no quadro sintomático e em suas percepções, acreditando que já não é mais necessário continuar com um tratamento tão prolongado já que não está sentindo mais nada (SILVA et al., 2010). Esse é um dos fatores contribuintes para o abandono do tratamento, uma vez que os portadores não tem consciência que é necessário realizar o tratamento completo e que só o médico poderá dar alta por cura. Tanto o portador quanto os familiares devem ser orientados pelos profissionais de saúde sobre os cuidados que devem adotar, assim como o esquema de tratamento, os efeitos adversos, a duração do tratamento e as consequências do tratamento, deixando sempre claro que é importante concluir o tratamento (FREITAS et al., 2012).

No acompanhamento dos portadores de TB incluem cuidados no tratamento que vão além das recomendações médicas, tais como: evitar o contato constante com poeiras e evitar lugares fechados; a representação que o tratamento de tuberculose é difícil está ligada nas sensações desagradáveis que os medicamentos causam, e é preciso empenho para conseguir realizá-lo (FARIAS et al., 2013).

\subsection{IMPORTÂNCIA DO CONTEXTO FAMILIAR PARA A ADESÃO À TERAPÊUTICA NA TUBERCULOSE}

A família é o alicerce fundamental para a continuidade do tratamento do portador de tuberculose (NOGUEIRA et al., 2011; OLIVEIRA et al., 2011), principalmente no apoio e acolhimento para evitar 0 abandono do tratamento. No presente estudo os entrevistados relatam a importância do apoio familiar frente ao diagnóstico e a força que transmitem para o tratamento.

[...] Quando fui diagnosticado, minha família não sabia, mais o médico conversou com eles e explicou que eu posso ficar boa, então eles me apoiaram. (BEIJA - FLOR).

[...] Tenho apoio. No início ficaram preocupados mais agora sabem que posso ficar bom. (CANÁRIO).

[...] A minha família apoiou, porque meu pai já tinha. (AZULÃO).
A importância da presença das pessoas que integram o lar pode ser um fator decisivo para a continuidade do tratamento, por que o portador terá com quem compartilhar seus medos e dificuldades inerentes ao tratamento medicamentoso, pois mesmo que o portador venha a ter vontade de interromper o tratamento à família estará junto dando apoio e forças para a não desistência (CLEMENTINO et al., 2011; OLIVEIRA et al., 2011). 0 âmbito familiar é primordial para a finalização do tratamento, pois influencia na adoção de novos hábitos de vida e condutas que favorecerão o êxito terapêutico (FREITAS et al., 2012).

A família e o portador de tuberculose devem estar cientes que a prioridade após o diagnóstico é realizar o tratamento adequado para evitar resistência medicamentosa ou reincidivas e obter a cura. 0 núcleo familiar no que diz respeito ao doente pode assumir dois papéis centrais na vida do portador de tuberculose, poderá ser a fonte de suporte e apoio no enfrentamento da doença e incentivá-lo a concluir o tratamento ou até levá-lo ao abandono do tratamento pelo isolamento decorrente da patologia (SOUSA; SILVA, 2013), pois existem diversos modos de o portador olhar e encarar a doença, contudo a família tem papel relevante no processo saúde/doença e na forma de tratá-la.

\subsection{O REFLEXO DO PRECONCEITO SOCIAL NA VIDA DO PORTADOR DE TUBERCULOSE}

A tuberculose é uma doença que tem tratamento e cura, com terapêutica farmacológica eficaz e medidas preventivas resolutivas (BRASIL, 2011; PINHEIRO et al., 2013; SILVA et al., 2016). Porém, é uma enfermidade que está envolta de tabus e crenças de natureza simbólica, advindas de várias épocas remotas e dos mais diferentes povos, hoje apesar de avanços científicos e haver tratamentos disponíveis gratuitamente e eficazes, o estigma em relação à doença acabam levando as pessoas que adquirem a tuberculose a sofrerem não só pelos sintomas clínicos, mas também já veem na mente a possibilidade de vivenciar os preconceitos e serem rejeitados no ambiente social e profissional (FARIAS et al., 2013). Na vivência dos portado- 
res em estudo não foi diferente, todos apresentaram algum sinal de preconceito e rejeição.

[...] Das pessoas da rua que sabiam que eu estava com tuberculose. (BEIJA - FLOR).

[...] Tive preconceito no ambiente de trabalho e com os amigos. (CANÁRIO).

[...] A vizinhança tem preconceito, porque tem medo de pegar a doença. (BEM TI VI).

[...] Os amigos, eu sei que tiveram porque alguns se afastaram (AZULÃO).

Mediante as situações de preconceito que acabam levando muitos portadores de TB a esconderem de seus ciclos de amizade e trabalho o seu estado de saúde, gerando assim dentro de si, anseios, medo e vergonha, dificultando a procura de tratamento e prolongando os riscos de disseminação dos bacilos no meio ambiente. A tuberculose é um dos problemas sociais que gera mudanças negativas como afastamento e isolamento na vida pessoal (CLEMENTINO et al., 2011).

0 temor de ser rejeitado perpassa pela mente de quase todos os portadores, por isso é importante que a família esteja presente desde o diagnóstico até a cura, pois é nela que o portador irá encontrar estímulo de vencer a doença. 0 afastamento de familiares e amigos acarreta na vida do portador sentimentos de pânico em relação à doença, é um acontecimento muitas vezes estressante que ameaça a vida do portador e seu isolamento no mundo (ROCHA; ADORNO, 2012). Contudo, estudos relatam que na atualidade, com o advento do tratamento eficaz, o portador de tuberculose já não é mais visto pela sociedade como perigo coletivo (ROCHA; CRUZ; FERMINO, 2013).

$O$ preconceito além de contribuir para o isolamento social, ainda faz com que o portador se distancie dos filhos e cônjuges, em alguns casos o preconceito acontece não somente advindos dos outros em relação à pessoa com tuberculose, mas da própria pessoa mergulhada nos próprios conceitos e pensamentos muitas vezes equivocados (FREITAS et al., 2012; SANTOS et al., 2014).
Desta forma, emerge a necessidade da equipe atuante na Atenção Básica (AB), demonstrar mais acolhimento ao tuberculínico, amenizando o preconceito, por meio de atividades educativas na comunidade. Um estudo realizado no Rio Grande do Norte, junto aos profissionais atuantes na $A B$, demonstrou que em relação a prática de atividades educativas e de promoção à saúde direcionadas para a TB, parte dos profissionais realizam tais atividades, em contrapartida, outros profissionais relataram que a equipe promove atividades com grupos de intervenção educacional, focando a orientação sobre doenças transmissíveis, entre elas a TB, conforme a necessidade do território (BRITO et al., 2015).

Os portadores de tuberculose precisam que os serviços de saúde foquem a busca ativa dos sintomáticos respiratórios e auxiliem no combate desta doença que já era para ter sido erradicada. Adicionalmente, deve-se promover educação em saúde para familiares e esclarecimento sobre a enfermidade para a sociedade, a fim de minimizar os preconceitos tão presentes na vida dos portadores.

\section{CONCLUSÃO}

Os resultados obtidos neste estudo evidenciaram as vivências de portadores de tuberculose, bem como a importância do apoio familiar e social no que diz respeito ao diagnóstico e tratamento na vida dos portadores de tuberculose. Viu-se que adoecer por uma patologia que além de debilitar o corpo ainda fragiliza o ser psicologicamente e socialmente não é fácil, devido à repercussão que a doença traz nos aspectos importantes de sua vida, gerando dentro de si conflitos, medos, angústias, afastamento do trabalho, mudanças de hábitos de vida, preconceito, restrição no convívio familiar e social, repercutindo negativamente sobre sua qualidade de vida.

0 cuidar em família é primordial para a adesão ao tratamento, uma vez que o portador de tuberculose encontrará no âmbito familiar o apoio necessário para dar continuidade ao mesmo e obter a cura. 
Percebeu-se que o preconceito social está muito presente nos relatos dos portadores, então se faz necessária uma abordagem mais clara pelos profissionais de saúde, de falar como é a transmissão da doença, o período de transmissibilidade e como deverá ser realizado o tratamento, deixar claro os efeitos adversos que as medicações causam e orientar as famílias, o portador e a sociedade que a tuberculose tem cura, tentando minimizar o preconceito ainda existente sobre a doença.

\section{REFERÊNCIAS}

BARDIN, L. Análise de conteúdo. São Paulo: 70, 2011. 229p. Disponível em: <http: http://www. reveduc.ufscar.br/index.php/reveduc/article/ view/291/156>. Acesso em: 6 jun. 2017

BRASIL. Ministério da Saúde. Resolução CNS No466, de 12 de dezembro de 2012. Brasília: Ministério da Saúde, 2013. Disponível em: <http: http:// bvsms.saude.gov.br/bvs/saudelegis/cns/2013/ res0466_12_12_2012.html>. Acesso em: 15 nov. 2015.

BRASIL. Tuberculose na atenção primária de saúde. Programa Nacional de Controle da Tuberculose. Brasília: MS, 2011. Disponível em: <http: http:// bvsms.saude.gov.br/bvs/publicacoes/manual_ recomendacoes_controle_tuberculose_brasil.pdf $>$. Acesso em: 20 out. 2015.

BRITO, E.W.G. et al. Care organization for tuberculosis in the primary care of Rio Grande do Norte. J Nurs UFPE on-line, v.9, n.6, p.864352, 2015. DOI: 10.5205/reuol.7061-61015-5SM0906supl201503. Acesso em: 15 nov. 2015.

CLEMENTINO, F.S. et al. Tuberculose: desvendando conflitos pessoais e sociais. Rev. Enferm. UERJ, v.19, n.4, p.638-43, 2011. Disponível em: <http: http://www.facenf.uerj.br/v19n4/v19n4a23.pdf>. Acesso em: 10 mar. 2015.
FARIAS, S.N.P. et al. Integralidade no cuidado: Estudo da qualidade de vida dos usuários com tuberculose.

Escola Anna Nery, v.17, n.4, p.749-54, 2013.

Disponível em: <http: http://eean.edu.br/detalhe artigo.asp?id=913>. Acesso em: 14 abr. 2015.

FREITAS, I.M. et al. Conhecimento e percepção sobre tuberculose das famílias de pacientes em tratamento diretamente observado em um serviço de saúde de Ribeirão Preto-SP, Brasil. Texto Contexto Enferm., v.21, n.3, p.642-9, 2012. Disponível em: <http: http:// www.scielo.br/pdf/tce/v21n3/v21n3a20>. Acesso em: 14 abr. 2015.

HIJAR, M.A.; CAMPOS, H.S.; FEITOSA, J.V.P. Tuberculose. In: COURA, J.R. Dinâmica das doenças infecciosas e parasitárias. Rio de Janeiro: Guanabara Koogan, 2013. p.1424-63.

LACERDA, S.N.B. et al. Individual and social vulnerabilities upon acquiring tuberculosis: a literature systematic review. Int. Arch. Med., v.7, n.35, p.1-8, 2014. DOI: 10.3823/1805. Acesso em: 16 mai. 2015.

LEMOS, L.A.; FEIJÃO, A.R.; GALVÃO, M.T.G. Aspectos sociais e de saúde de portadores da coinfecção HIV/ tuberculose. RevRENE, v.14, n.2, p.364-71, 2013. Disponível em: <http: http://www.repositorio.ufc.br/ handle/riufc/7132> Acesso em: 16 maio 2015.

MACIEL, M.S. A história da tuberculose no Brasil: os muitos tons (de cinza) da miséria. Rev.

Bras. Clin. Méd., São Paulo, v.10, n.3, p.226-30, 2012. Disponivel em: <http: http://files.bvs.br/ upload/S/1679-1010/2012/v10n3/a2886.pdf>. Acesso em: 16 maio 2015.

MARQUIEVIZ, J. et al. A Estratégia de Saúde da Família no controle da tuberculose em Curitiba (PR). Ciên. Saúde Col., v.18, n.1, p.265-271, 2013. Disponível em: <http: www.scielo.br/pdf/csc/ v18n1/27.pdf>. Acesso em: 20 abr. 2015. 
NOGUEIRA, J.A. et al. Enfoque familiar e orientação para a comunidade no controle da tuberculose. Rev. Brasil. Epidemiol., v.14, n.2, p.207-216, 2011. Disponível em: <http: http:// www.scielo.br/pdf/rbepid/v14n2/03.pdf>. Acesso em: 15 fev. 2015.

\section{OLIVEIRA, M.G. et al. 0 doente em tratamento} de tuberculose no município de Itaboraí, Rio de Janeiro - participação da família. Rev. Bras. Med. Família Com., v.6, n.18, p.40-45, 2011. Disponível em: <http: https://rbmfc.org.br/rbmfc/article/ view/117>. Acesso em: 15 nov. 2015.

PINHEIRO, R.S. et al. Determinantes sociais e auto relato de tuberculose nas regiões metropolitanas conforme a pesquisa nacional por amostras em domicílios, Brasil. Rev Panam Salud Publica, v.34, n.6, p.446-451, 2013. Disponível em: <http: www. scielosp.org/pdf/rpsp/v34n6/v34n6a11.pdf>. Acesso em: 17 nov. 2015.

ROCHA, A.; CRUZ, J.A.; FERMINO, S.M.D.A. Tuberculose pulmonar: conhecer para melhor cuidar. Portal do conhecimento. 2013.61f. Monografia (Graduação em Enfermagem) Universidade do Mindelo, Mindelo, 2013.

\section{ROCHA, D.S.; ADORNO, R.C.F. Dropouts or} discontinuity of the treatment of tuberculosis in Rio Branco, Acre. Soc Salud., v.21, n.1, p.232-45, 2012. Disponível em: <http://www.producao.usp. br/bitstream/handle/BDPI/34497/wos2012-5889_ pt.pdf?sequence=1. Acesso em: 20 nov. 2015.
SANTOS, E.F.S.S. et al. Caráter estigmatizante da tuberculose, natureza biológica e impacto social da doença. Rev. Faculdade Odontol. Lins, v.24, n.1, p.41-50, 2014. Disponível em: <http: https://www. metodista.br/revistas/revistas-unimep/index.php/Fol/ article/view/1934/1335>. Acesso em: 15 nov. 2015.

SILVA, A.M.F.B. et al. O corpo do portador de tuberculose: Enfrentamentos, Dificuldades e Projeções diárias na terapia ocupacional para obtenção da cura. Rev. Pesq: cuidado é fundamental online, v.2, n.4, p.1197-13, 2010. Disponível em: <http: saudepublica.bvs.br/pesquisa/resource/pt/ bde-22169>. Acesso em: 15 nov. 2015.

SILVA, C.S. et al. Frequência de casos positivos para tuberculose em Tobias Barreto-SE no período de 2010-2015. Interf. Cient. Saúde Amb., v.4, n.2, p.2934, 2016. Disponível em: <http: https://periodicos. set.edu.br/index.php/saude/article/download/3004/ pdf_14>. Acesso em: 15 nov. 2015.

SOUSA, M.N.A.; SILVA, J.D.F. Práticas do autocuidado entre os portadores de tuberculose de município paraibano. C\&D - Rev. Eletr. da Fainor, v.6, n.2, p.150-161, 2013. Disponível em: <http: http://srv02. fainor.com.br/revista/index.php/memorias/article/ view/229>. Acesso em: 15 nov. 2015.

SOUSA, S.S.; SILVA, D.M.G.V.; MEIRELLES, B.H.S. Representações sociais sobre a tuberculose. Acta Paul Enferm., v.23, n.1, p.23-8. 2010. Disponível em: <http: www.scielo.br/pdf/ape/v23n1/04.pdf>. Acesso em: 15 nov. 2015. 